\title{
Qualitative and quantitative analysis for solutions to a class of Volterra-Fredholm type difference equation
}

\author{
Bin Zheng
}

Correspondence:

zhengbin2601@126.com

School of Science, Shandong

University of Technology,

Zhangzhou Road 12, Zibo,

Shandong, 255049, China

\section{Springer}

\section{Abstract}

In this paper, we present some new discrete Volterra-Fredholm type inequalities, based on which we study the qualitative and quantitative properties of solutions of a class of Volterra-Fredholm type difference equation. Some results on the boundedness, uniqueness, and continuous dependence on initial data of solutions are established under some suitable conditions.

Mathematics Subject Classification 2010: 26D15

Keywords: discrete inequalities, Volterra-Fredholm type difference equations, qualitative analysis, quantitative analysis, bounded

\section{Introduction}

In this paper, we study a class of Volterra-Fredholm type difference equation with the following form

$$
\begin{aligned}
z^{p}(m, n)= & g_{1}(m, n)+\sum_{s=m+1}^{\infty} g_{2}(s, n) z^{p}(s, n) \\
& +\sum_{i=1}^{l_{1}} \sum_{s=m+1}^{\infty} \sum_{t=n+1}^{\infty}\left[F_{1 i}(s, t, m, n, z(s, t))+\sum_{\xi=s}^{\infty} \sum_{\eta=t}^{\infty} F_{2 i}(\xi, \eta, m, n, z(\xi, \eta))\right] \\
& +\sum_{i=1}^{l_{2}} \sum_{s=M+1}^{\infty} \sum_{t=N+1}^{\infty}\left[G_{1 i}(s, t, m, n, z(s, t))+\sum_{\xi=s}^{\infty} \sum_{\eta=t}^{\infty} G_{2 i}(\xi, \eta, m, n, z(\xi, \eta))\right],
\end{aligned}
$$

where $z(m, n), g_{1}(m, n), g_{2}(m, n)$ are $\mathbb{R}$-valued functions defined on $\Omega, F_{1 i}, F_{2 i}, i=1$, $2, \ldots, l_{2}$ and $G_{1 i}, G_{2 i}, i=1,2, \ldots, l_{2}$ are $\mathbb{R}$-valued functions defined on $\Omega^{2} \times \mathbb{R}, \mathbf{p} \geq \mathbf{1}$ is an odd number.

Volterra-Fredholm type difference equations can be considered as the discrete analog of classical Volterra-Fredholm type integral equations, which arise in the theory of parabolic boundary value problems, the mathematical modeling of the spatio-temporal development of an epidemic, and various physical and biological problems. For Eq. (1), if we take $l_{1}=l_{2}=1, F_{21}(\xi, \eta, m, n, z(\xi, \eta))=G_{21}(\xi, \eta, m, n, z(\xi, \eta)) \equiv 0$, then Eq. (1) becomes the discrete version with infinite sum upper limit of [[1], Eq. (3.1)]. Some concrete forms of Eq. (1) are also variations of some known difference equations in the literature to infinite sum upper limit. For example, If $l_{1}=l_{2}=1, F_{21}(\xi, \eta, m, n, z(\xi, \eta))$ 
$=G_{21}(\xi, \eta, m, n, z(\xi, \eta)) \equiv 0$, then Eq. (1) becomes the variation of [[2,3], Eq. (3.1)]. If $l_{1}=l_{2}=1, F_{1}(s, t, m, n, z(s, t))=F(s, t, m, n, z(s, t))+H(s, t, m, n, z(s, t)), F_{21}(\xi, \eta$, $m, n, z(\xi, \eta))=G_{11}(s, t, m, n, z(s, t))=G_{21}(\xi, \eta, m, n, z(\xi, \eta)) \equiv 0$, then Eq. (1) becomes the variation of [[4], Eq. (4.1)].

In the research of solutions of certain difference equations, if the solutions are unknown, then it is necessary to study their qualitative and quantitative properties such as boundedness, uniqueness, and continuous dependence on initial data. The Gronwall-Bellman inequality $[5,6]$ and its various generalizations that provide explicit bounds play a fundamental role in the research of this domain. Many such generalized inequalities (for example, see [7-16] and the references therein) have been established in the literature including the known Ou-lang's inequality [7], which provide handy tools in the study of qualitative and quantitative properties of solutions of certain difference equations. In [2], Ma generalized the discrete version of Ou-lang's inequality in two variables to Volterra-Fredholm form for the first time, which has proved to be very useful in the study of properties of solutions of certain Volterra-Fredholm type difference equations. But since then, few results on Volterra-Fredholm type discrete inequalities have been established. Recent result in this direction only includes the work of $\mathrm{Ma}$ [3] to our knowledge. We note in order to fulfill the analysis of qualitative and quantitative properties of the solutions of Eq. (1), which has more complicated form than the example presented in [3], the results provided by the earlier inequalities are inadequate and it is necessary to seek some new Volterra-Fredholm type discrete inequalities so as to obtain desired results.

This paper is organized as follows. First, we establish some new Volterra-Fredholm type discrete inequalities, based on which we derive explicit bounds for the solutions of Eq. (1) under some suitable conditions. Then, some results about the uniqueness and continuous dependence on the functions $g_{1}, F_{1 i}, F_{2 i}, G_{1 i}, G_{2 i}$ of the solutions of Eq. (1) are established using the presented inequalities.

Throughout this paper, $\mathbb{R}$ denotes the set of real numbers and $\mathbb{R}_{+}=[0, \infty)$, while $\mathbb{Z}$ denotes the set of integers. Let $\Omega:=([M, \infty] \times[N, \infty]) \cap \mathbb{Z}^{2}$, where $M, N \in \mathbb{Z}$ are two constants. $p \geq 1$ is an odd number. $l_{1}, l_{2} \in \mathbb{Z}, K_{i} \in \mathbb{R}, i=1,2,3,4$ are constants with $l_{1} \geq 1, l_{2} \geq 1, K_{i}>0$. If $U$ is a lattice, then we denote the set of all $\mathbb{R}$-valued functions on $U$ by $\wp(U)$, and denote the set of all $\mathbb{R}_{+}$-valued functions on $U$ by $\wp_{+}(U)$. As usual, the collection of all continuous functions of a topological space $X$ into another topological space $Y$ is denoted by $C(X, Y)$. Finally, for a $\mathbb{R}_{+}$-valued function such as $f \in \wp$ + $(\Omega)$, we note $\sum_{s=m_{0}}^{m_{1}} f(s, n)=0$ provided $m_{0}>m_{1}$, and $\lim _{m \rightarrow \infty} \sum_{s=m+1}^{\infty} f(s, n)=0$.

\section{Some new Volterra-Fredholm type discrete inequalities}

Lemma 2:1. Suppose $u(m, n), a(m, n), b(m, n) \in \wp_{+}(\Omega)$. If $a(m, n)$ is nonincreasing in the first variable, then for $(m, n) \in \Omega$,

$$
u(m, n) \leq a(m, n)+\sum_{s=m+1}^{\infty} b(s, n) u(s, n)
$$


implies

$$
u(m, n) \leq a(m, n) \prod_{s=m+1}^{\infty}[1+b(s, n)]
$$

Remark 1. Lemma 2.1 is a direct variation of [[13], Lemma $\left.2.5\left(\beta_{2}\right)\right]$, and we note $a$ $(m, n) \geq 0$ here.

Lemma 2.2. Suppose $u(m, n), a(m, n) \in \wp_{+}(\Omega), b(s, t, m, n) \in \wp_{+}\left(\Omega^{2}\right)$, and $a(m, n)$ is nonincreasing in every variable with $a(m, n)>0$, while $b(s, t, m, n)$ is nonincreasing in the third variable. $\phi \in C\left(\mathbb{R}_{+}, \mathbb{R}_{+}\right)$is nondecreasing with $\phi(r)>0$ for $r>0$. If for $(m$, $n) \in \Omega, u(m, n)$ satisfies the following inequality

$$
u(m, n) \leq a(m, n)+\sum_{s=m+1}^{\infty} \sum_{t=n+1}^{\infty} b(s, t, m, n) \varphi\left(u^{\frac{1}{p}}(s, t)\right),
$$

then we have

$$
u(m, n) \leq G^{-1}\left[G(a(m, n))+\sum_{s=m+1}^{\infty} \sum_{t=n+1}^{\infty} b(s, t, m, n)\right]
$$

where

$$
G(z)=\int_{z_{0}}^{z} \frac{1}{\varphi\left(z^{\frac{1}{p}}\right)} \mathrm{d} z, z \geq z_{0}>0 .
$$

Proof. Fix $\left(m_{1}, n_{1}\right) \in \Omega$, and let $(m, n) \in\left(\left[m_{1}, \infty\right] \times\left[n_{1}, \infty\right]\right) \cap \Omega$. Then, we have

$$
u(m, n) \leq a\left(m_{1}, n_{1}\right)+\sum_{s=m+1}^{\infty} \sum_{t=n+1}^{\infty} b(s, t, m, n) \varphi\left(u^{\frac{1}{p}}(s, t)\right) .
$$

Let the right side of (5) be $v(m, n)$. Then,

$$
u(m, n) \leq v(m, n),(m, n) \in\left(\left[m_{1}, \infty\right] \times\left[n_{1}, \infty\right]\right) \bigcap \Omega,
$$

and

$$
\begin{aligned}
v(m-1, n)-v(m, n)= & \sum_{s=m}^{\infty} \sum_{t=n+1}^{\infty} b(s, t, m-1, n) \varphi\left(u^{\frac{1}{p}}(s, t)\right)-\sum_{s=m+1}^{\infty} \sum_{t=n+1}^{\infty} b(s, t, m, n) \varphi\left(u^{\frac{1}{p}}(s, t)\right) \\
= & \sum_{s=m}^{\infty} \sum_{t=n+1}^{\infty} b(s, t, m-1, n) \varphi\left(u^{\frac{1}{p}}(s, t)\right)-\sum_{s=m+1}^{\infty} \sum_{t=n+1}^{\infty} b(s, t, m-1, n) \varphi\left(u^{\frac{1}{p}}(s, t)\right) \\
& +\sum_{s=m+1}^{\infty} \sum_{t=n+1}^{\infty} b(s, t, m-1, n) \varphi\left(u^{\frac{1}{p}}(s, t)\right)-\sum_{s=m+1}^{\infty} \sum_{t=n+1}^{\infty} b(s, t, m, n) \varphi\left(u^{\frac{1}{p}}(s, t)\right) \\
= & \sum_{t=n+1}^{\infty} b(m, t, m-1, n) \varphi\left(u^{\frac{1}{p}}(m, t)\right)+\sum_{s=m+1}^{\infty} \sum_{t=n+1}^{\infty}[b(s, t, m-1, n)-b(s, t, m, n)] \varphi\left(u^{\frac{1}{p}}(s, t)\right) \\
\leq & \sum_{t=n+1}^{\infty} b(m, t, m-1, n) \varphi\left(v^{\frac{1}{p}}(m, t)\right)+\sum_{s=m+1}^{\infty} \sum_{t=n+1}^{\infty}[b(s, t, m-1, n)-b(s, t, m, n)] \varphi\left(v^{\frac{1}{p}}(s, t)\right) \\
\leq & \left\{\sum_{t=n+1}^{\infty} b(m, t, m-1, n)+\sum_{s=m+1}^{\infty} \sum_{t=n+1}^{\infty}[b(s, t, m-1, n)-b(s, t, m, n)]\right\} \varphi\left(v^{\frac{1}{p}}(m, n)\right),
\end{aligned}
$$


that is,

$$
\begin{aligned}
& \frac{v(m-1, n)-v(m, n)}{\varphi\left(v^{\frac{1}{p}}(m, n)\right)} \leq \sum_{t=n+1}^{\infty} b(m, t, m-1, n)+\sum_{s=m+1}^{\infty} \sum_{t=n+1}^{\infty}[b(s, t, m-1, n)-b(s, t, m, n)] \\
& =\sum_{s=m}^{\infty} \sum_{t=n+1}^{\infty} b(s, t, m-1, n)-\sum_{s=m+1}^{\infty} \sum_{t=n+1}^{\infty} b(s, t, m-1, n)+\sum_{s=m+1}^{\infty} \sum_{t=n+1}^{\infty}[b(s, t, m-1, n)-b(s, t, m, n)] \\
& =\sum_{s=m}^{\infty} \sum_{t=n+1}^{\infty} b(s, t, m-1, n)-\sum_{s=m+1}^{\infty} \sum_{t=n+1}^{\infty} b(s, t, m, n) .
\end{aligned}
$$

On the other hand, according to the Mean Value Theorem for integrals, there exists $\xi$ such that $v(m, n) \leq \xi \leq v(m-1, n)$, and

$$
\int_{v(m, n)}^{v(m-1, n)} \frac{1}{\varphi\left(z^{\frac{1}{p}}\right)} d z=\frac{v(m-1, n)-v(m, n)}{\varphi\left(\xi^{\frac{1}{p}}\right)} \leq \frac{v(m-1, n)-v(m, n)}{\varphi\left(v^{\frac{1}{p}}(m, n)\right)} .
$$

So, combining (7) and (8), we have

$$
\begin{aligned}
\int_{v(m, n)}^{v(m-1, n)} \frac{1}{\varphi\left(z^{\frac{1}{p}}\right)} \mathrm{d} z & =G(v(m-1, n))-G(v(m, n)) \\
& \leq \sum_{s=m}^{\infty} \sum_{t=n+1}^{\infty} b(s, t, m-1, n)-\sum_{s=m+1}^{\infty} \sum_{t=n+1}^{\infty} b(s, t, m, n),
\end{aligned}
$$

where $G$ is defined in (4). Setting $m=\eta$ in (9), and a summary with respect to $\eta$ from $m+1$ to $\infty$ yields

$$
G(v(m, n))-G(v(\infty, n)) \leq \sum_{s=m+1}^{\infty} \sum_{t=n+1}^{\infty} b(s, t, m, n)-0=\sum_{s=m+1}^{\infty} \sum_{t=n+1}^{\infty} b(s, t, m, n) .
$$

Noticing $v(\infty, n)=a\left(m_{1}, n_{1}\right)$, and $G$ is increasing, it follows

$$
v(m, n) \leq G^{-1}\left[G\left(a\left(m_{1}, n_{1}\right)\right)+\sum_{s=m+1}^{\infty} \sum_{t=n+1}^{\infty} b(s, t, m, n)\right] .
$$

Combining (6) and (10), we obtain

$$
u(m, n) \leq G^{-1}\left[G\left(a\left(m_{1}, n_{1}\right)\right)+\sum_{s=m+1}^{\infty} \sum_{t=n+1}^{\infty} b(s, t, m, n)\right],(m, n) \in\left(\left[m_{1}, \infty\right] \times\left[n_{1}, \infty\right]\right) \bigcap \Omega .
$$

Setting $m=m_{1}, n=n_{1}$ in (11), yields

$$
u\left(m_{1}, n_{1}\right) \leq G^{-1}\left[G\left(a\left(m_{1}, n_{1}\right)\right)+\sum_{s=m_{1}+1}^{\infty} \sum_{t=n_{1}+1}^{\infty} b\left(s, t, m_{1}, n_{1}\right)\right] .
$$

Since $\left(m_{1}, n_{1}\right)$ is selected from $\Omega$ arbitrarily, then substituting $\left(m_{1}, n_{1}\right)$ with $(m, n)$ in (12), we get the desired inequality (3).

Corollary 2. 3. Under the conditions of Lemma 2.2, and furthermore assume $a(m, n)$ $\geq 0$. If for $(m, n) \in \Omega, u(m, n)$ satisfies the following inequality

$$
u(m, n) \leq a(m, n)+\sum_{s=m+1}^{\infty} \sum_{t=n+1}^{\infty} b(s, t, m, n) u(s, t)
$$


then we have

$$
u(m, n) \leq a(m, n) \exp \left\{\sum_{s=m+1}^{\infty} \sum_{t=n+1}^{\infty} b(s, t, m, n)\right\},
$$

Proof. Suppose $a(m, n)>0$. By Theorem 2.1 (with $\varphi\left(u^{\frac{1}{p}}\right)=1$ ), we have

$$
u(m, n) \leq G^{-1}\left[G(a(m, n))+\exp \left\{\sum_{s=m+1}^{\infty} \sum_{t=n+1}^{\infty} b(s, t, m, n)\right\}\right]
$$

Where $G(z)=\int_{z_{0}}^{z} \frac{1}{z} \mathrm{~d} z=\ln z-\ln z_{0}, z \geq z_{0}>0$. Then, a simplification of (15) yields the desired inequality (14).

If $a(m, n) \geq 0$, then we can carry out the process above with $a(m, n)$ replaced by $a$ $(m, n)+\varepsilon$, where $\varepsilon>0$. After letting $\varepsilon \rightarrow 0$, we also obtain the desired inequality (14).

Lemma 2.4 [[17]]. Assume that $a \geq 0, p \geq q \geq 0$, and $p \neq 0$, then for any $K>0$

$$
a^{\frac{q}{p}} \leq \frac{q}{p} K^{\frac{q-p}{p}} a+\frac{p-q}{p} K^{\frac{q}{p}}
$$

Theorem 2:5. Suppose, $u(m, n), w(m, n) \in \wp_{+}(\Omega), b_{i}(s, t, m, n), c_{i}(s, t, m, n) \in \wp$ ${ }_{+}\left(\Omega^{2}\right), i=1,2, \ldots, l_{1}, d_{i}(s, t, m, n), e_{i}(s, t, m, n) \in \wp_{+}\left(\Omega^{2}\right), i=1,2, \ldots, l_{2}$ with $b_{i}, c_{i}, d_{i}, e_{i}$ nonincreasing in the last two variables, and there is at least one function among $d_{i}, e_{i}$, $i=1,2, \ldots, l_{2}$ not equivalent to zero. $\phi \in C\left(\mathbb{R}_{+}, \mathbb{R}_{+}\right)$is nondecreasing with $\phi(r)>0$ for $r$ $>0$, and $\phi$ is submultiplicative, that is, $\phi(\alpha \beta) \leq \phi(\alpha) \phi(\beta)$ for $\forall \alpha, \beta \in \mathbb{R}_{+}$. If for $(m, n)$ $\in \Omega, u(m, n)$ satisfies the following inequality

$$
\begin{aligned}
u^{p}(m, n) & \leq \sum_{s=m+1}^{\infty} w(s, n) u^{p}(s, n)+\sum_{i=1}^{l_{1}} \sum_{s=m+1}^{\infty} \sum_{t=n+1}^{\infty}\left[b_{i}(s, t, m, n) \varphi(u(s, t))\right. \\
& \left.+\sum_{\xi=s}^{\infty} \sum_{\eta=t}^{\infty} c_{i}(\xi, \eta, m, n) \varphi(u(\xi, \eta))\right]+\sum_{i=1}^{l_{2}} \sum_{s=M+1}^{\infty} \sum_{t=N+1}^{\infty}\left[d_{i}(s, t, m, n) u^{p}(s, t)\right. \\
& \left.+\sum_{\xi=s}^{\infty} \sum_{\eta=t}^{\infty} e_{i}(\xi, \eta, m, n) u^{p}(\xi, \eta)\right]
\end{aligned}
$$

then we have

$$
u(m, n) \leq\left\{G^{-1}\left[G\left(J^{-1}(C(M, N))\right)+C(m, n)\right] \bar{w}(m, n)\right\}^{\frac{1}{p}},
$$

provided that $0<\mu<1$ and $J$ is increasing, where

$$
\begin{aligned}
& G(z)=\int_{z_{0}}^{z} \frac{1}{\varphi\left(z^{\frac{1}{p}}\right)} \mathrm{d} z, z \geq z_{0}>0, \\
& J(x)=G\left(\frac{x}{\mu}\right)-G(x), x \geq 0, \\
& \bar{w}(m, n)=\prod_{s=m+1}^{\infty}[1+w(s, n)]
\end{aligned}
$$




$$
\begin{aligned}
& C(m, n)=\sum_{s=m+1}^{\infty} \sum_{t=n+1}^{\infty} B(s, t, m, n), \\
& B(s, t, m, n)=\sum_{i=1}^{l_{1}}\left[b_{i}(s, t, m, n) \varphi\left(\bar{w}^{\frac{1}{p}}(s, t)\right)+\sum_{\xi=s}^{\infty} \sum_{\eta=t}^{\infty} c_{i}(\xi, \eta, m, n) \phi\left(\bar{w}^{\frac{1}{p}}(\xi, \eta)\right)\right], \\
& \mu=\sum_{i=1}^{l_{2}} \sum_{s=M+1}^{\infty} \sum_{t=N+1}^{\infty}\left[d_{i}(s, t, M, N) \bar{w}(s, t)+\sum_{\xi=s}^{\infty} \sum_{\eta=t}^{\infty} e_{i}(\xi, \eta, M, N) \bar{w}(\xi, \eta)\right] .
\end{aligned}
$$

Proof. Denote the right side of (16) be $v(m, n)+\sum_{s=m+1}^{\infty} w(s, n) u^{p}(s, n)$. Then, $v(m, n)$ is nonincreasing in every variable, and by Lemma 2.1 , we obtain

$$
u^{p}(m, n) \leq v(m, n) \prod_{s=m+1}^{\infty}[1+w(s, n)]=v(m, n) \bar{w}(m, n),
$$

where $\bar{w}(m, n)$ is defined in (20). Furthermore, by (24), we deduce

$$
\begin{aligned}
v(m, n) \leq & \sum_{i=1}^{l_{1}} \sum_{s=m+1}^{\infty} \sum_{t=n+1}^{\infty}\left[b_{i}(s, t, m, n) \varphi\left(v^{\frac{1}{p}}(s, t) \bar{w}^{\frac{1}{p}}(s, t)\right)\right. \\
& \left.+\sum_{\xi=s}^{\infty} \sum_{\eta=t}^{\infty} c_{i}(\xi, \eta, m, n) \varphi\left(v^{\frac{1}{p}}(\xi, \eta) \bar{w}^{\frac{1}{p}}(\xi, \eta)\right)\right] \\
& +\sum_{i=1}^{l_{2}} \sum_{s=M+1}^{\infty} \sum_{t=N+1}^{\infty}\left[d_{i}(s, t, m, n) \bar{w}(s, t) v(s, t)+\sum_{\xi=s}^{\infty} \sum_{\eta=t}^{\infty} e_{i}(\xi, \eta, m, n) \bar{w}(\xi, \eta) v(\xi, \eta)\right] \\
\leq & \sum_{i=1}^{l_{1}} \sum_{s=m+1}^{\infty} \sum_{t=n+1}^{\infty}\left[b_{i}(s, t, m, n) \varphi\left(\bar{w}^{\frac{1}{p}}(s, t)\right) \varphi\left(v^{\frac{1}{p}}(s, t)\right)+\sum_{\xi=s}^{\infty} \sum_{\eta=t}^{\infty} c_{i}(\xi, \eta, m, n) \varphi\left(\bar{w}^{\frac{1}{p}}(\xi, \eta)\right) \varphi\left(v^{\frac{1}{p}}(\xi, \eta)\right)\right] \\
& +\sum_{i=1}^{l_{2}} \sum_{s=M+1}^{\infty} \sum_{t=N+1}^{\infty}\left[d_{i}(s, t, m, n) \bar{w}(s, t) v(s, t)+\sum_{\xi=s}^{\infty} \sum_{\eta=t}^{\infty} e_{i}(\xi, \eta, m, n) \bar{w}(\xi, \eta) v(\xi, \eta)\right] \\
\leq & \sum_{i=1}^{l_{1}} \sum_{s=m+1}^{\infty} \sum_{t=n+1}^{\infty}\left[b_{i}(s, t, m, n) \varphi\left(\bar{w}^{\frac{1}{p}}(s, t)\right)+\sum_{\xi=s}^{\infty} \sum_{\eta=t}^{\infty} c_{i}(\xi, \eta, m, n) \varphi\left(\bar{w}^{\frac{1}{p}}(\xi, \eta)\right)\right] \varphi\left(v^{\frac{1}{p}}(s, t)\right) \\
& +\sum_{i=1}^{l_{2}} \sum_{s=M+1}^{\infty} \sum_{t=N+1}^{\infty}\left[d_{i}(s, t, m, n) \bar{w}(s, t) v(s, t)+\sum_{\xi=s}^{\infty} \sum_{\eta=t}^{\infty} e_{i}(\xi, \eta, m, n) \bar{w}(\xi, \eta) v(\xi, \eta)\right] \\
= & H(m, n)+\sum_{s=m+1}^{\infty} \sum_{t=n+1}^{\infty} B(s, t, m, n) \varphi\left(v^{\frac{1}{p}}(s, t)\right),
\end{aligned}
$$

where $\quad H(m, n)=\sum_{i=1}^{l_{2}} \sum_{s=M+1}^{\infty} \sum_{t=N+1}^{\infty}\left[d_{i}(s, t, m, n) \bar{w}(s, t) v(s, t)+\sum_{\xi=s}^{\infty} \sum_{\eta=t}^{\infty} e_{i}(\xi, \eta, m, n) \bar{w}(\xi, \eta) v(\xi, \eta)\right]$, and $B(s, t, m, n)$ is defined in (22).

As we can see, $H(m, n)$ is nonincreasing in every variable. Considering $m \geq M, n \geq$ $N$, it follows

$$
v(m, n) \leq H(M, N)+\sum_{s=m+1}^{\infty} \sum_{t=n+1}^{\infty} B(s, t, m, n) \varphi\left(v^{\frac{1}{p}}(s, t)\right) .
$$

Since there is at least one function among $d_{i}, e_{i}, i=1,2, \ldots, l_{2}$ not equivalent to zero, then $H(M, N)>0$.

On the other hand, as $b_{i}(s, t, m, n), c_{i}(s, t, m, n)$ are nonincreasing in the last two variables, then one can see $B(s, t, m, n)$ is also nonincreasing in the last two variables. So, a suitable application of Lemma 2.2 yields 


$$
v(m, n) \leq G^{-1}\left[G(H(M, N))+\sum_{s=m+1}^{\infty} \sum_{t=n+1}^{\infty} B(s, t, m, n)\right]=G^{-1}[G(H(M, N))+C(m, n)],
$$

where $G, C(m, n)$ are defined in (18) and (21), respectively. On the other hand, we have

$$
H(M, N)=\sum_{i=1}^{l_{2}} \sum_{s=M+1}^{\infty} \sum_{t=N+1}^{\infty}\left[d_{i}(s, t, M, N) \bar{w}(s, t) v(s, t)+\sum_{\xi=s}^{\infty} \sum_{\eta=t}^{\infty} e_{i}(\xi, \eta, M, N) \bar{w}(\xi, \eta) v(\xi, \eta)\right] .
$$

Then, considering $v(m, n)$ is nonincreasing in every variable, using (25) in (26) yields

$$
\begin{aligned}
H(M, N) \leq & v(M, N) \sum_{i=1}^{l_{2}} \sum_{s=M+1}^{\infty} \sum_{t=N+1}^{\infty}\left[d_{i}(s, t, M, N) \bar{w}(s, t)+\sum_{\xi=s}^{\infty} \sum_{\eta=t}^{\infty} e_{i}(\xi, \eta, M, N) \bar{w}(\xi, \eta)\right] \\
\leq & G^{-1}[G(H(M, N))+C(M, N)] \sum_{i=1}^{l_{2}} \sum_{s=M+1}^{\infty} \sum_{t=N+1}^{\infty}\left[d_{i}(s, t, M, N) \bar{w}(s, t)\right. \\
& \left.+\sum_{\xi=s}^{\infty} \sum_{\eta=t}^{\infty} e_{i}(\xi, \eta, M, N) \bar{w}(\xi, \eta)\right]=\mu G^{-1}[G(H(M, N))+C(M, N)]
\end{aligned}
$$

where $\mu$ is a constant defined in (23).

According to $0<\mu<1$, and $G$ is increasing, we obtain

$$
\frac{H(M, N)}{\mu} \leq G^{-1}[G(H(M, N))+C(M, N)]
$$

and

$$
G\left(\frac{H(M, N)}{\mu}\right) \leq G(H(M, N))+C(M, N) .
$$

which is rewritten by

$$
J(H(M, N)) \leq C(M, N),
$$

where $J$ is defined in (19). Since $J$ is increasing, we have

$$
H(M, N) \leq J^{-1}(C(M, N)) .
$$

Combining (24), (25), and (27), we get the desired result.

Theorem 2.6. Suppose, $u(m, n), a(m, n), w(m, n) \in \wp_{+}(\Omega), b_{i}(s, t, m, n), c_{i}(s, t, m, n)$ $\in \wp_{+}\left(\Omega^{2}\right), i=1,2, \ldots, l_{1}, d_{i}(s, t, m, n), e_{i}(s, t, m, n) \in \wp_{+}\left(\Omega^{2}\right), i=1,2, \ldots, l_{2}$ with $b_{i}, c_{i}$, $d_{i}, e_{i}$ nonincreasing in the last two variables. $q_{i}, r_{i}$ are nonnegative constants with $p \geq$ $q_{i}, p \geq r_{i}, i=1,2, \ldots, l_{1}$, while $h_{i}, j_{i}$ are nonnegative constants with $p \geq h_{i}, p \geq j_{i}, i=1$, $2, \ldots, l_{2}$. If for $(m, n) \in \Omega, u(m, n)$ satisfies the following inequality

$$
\begin{aligned}
u^{p}(m, n) \leq & a(m, n)+\sum_{s=m+1}^{\infty} w(s, n) u^{p}(s, n) \\
& +\sum_{i=1}^{l_{1}} \sum_{s=m+1}^{\infty} \sum_{t=n+1}^{\infty}\left[b_{i}(s, t, m, n) u^{q_{i}}(s, t)+\sum_{\xi=m_{0}}^{s} \sum_{\eta=n_{0}}^{t} c_{i}(\xi, \eta, m, n) u^{r_{i}}(\xi, \eta)\right] \\
& +\sum_{i=1}^{l_{2}} \sum_{s=M+1}^{\infty} \sum_{t=N+1}^{\infty}\left[d_{i}(s, t, m, n) u^{h_{i}}(s, t)+\sum_{\xi=m_{0}}^{s} \sum_{\eta=n_{0}}^{t} e_{i}(\xi, \eta, m, n) u^{j_{i}}(\xi, \eta)\right],
\end{aligned}
$$


then

$$
u(m, n) \leq\left\{\left[a(m, n)+\frac{\tilde{J}(M, N)}{1-\tilde{\mu}} \tilde{C}(m, n)\right] \tilde{w}(m, n)\right\}^{\frac{1}{p}},
$$

provided that $\tilde{\mu}<1$, where

$$
\begin{aligned}
\tilde{J}(m, n)= & \sum_{i=1}^{l_{1}} \sum_{s=m+1}^{\infty} \sum_{t=n+1}^{\infty}\left\{\tilde{b}_{i}(s, t, m, n)\left[\frac{q_{i}}{p} K_{1}^{\frac{q_{i}-p}{p}} a(s, t)+\frac{p-q_{i}}{p} K_{1}^{\frac{q_{i}}{p}}\right]\right. \\
& \left.+\sum_{\xi=s}^{\infty} \sum_{\eta=t}^{\infty} \tilde{c}_{i}(\xi, \eta, m, n)\left[\frac{r_{i}}{p} K_{2}^{\frac{r_{i}-p}{p}} a(\xi, \eta)+\frac{p-r_{i}}{p} K_{2}^{\frac{r_{i}}{p}}\right]\right\} \\
& +\sum_{i=1}^{l_{2}} \sum_{s=M+1}^{\infty} \sum_{t=N+1}^{\infty}\left\{\tilde{d}_{i}(s, t, m, n)\left[\frac{h_{i}}{p} K_{3}^{\frac{h_{i}-p}{p}} a(s, t)+\frac{p-h_{i}}{p} K_{3}^{\frac{h_{i}}{p}}\right]\right. \\
& \left.+\sum_{\xi=s}^{\infty} \sum_{\eta=t}^{\infty} \tilde{e}_{i}(\xi, \eta, m, n)\left[\frac{j_{i}}{p} K_{4}^{\frac{j_{i}-p}{p}} a(\xi, \eta)+\frac{p-j_{i}}{p} K_{4}^{\frac{j_{i}}{p}}\right]\right\},
\end{aligned}
$$

$\tilde{b}_{i}(s, t, m, n)=b_{i}(s, t, m, n)(\tilde{w}(s, t))^{\frac{q_{i}}{p}}, \tilde{c}_{i}(s, t, m, n)=c_{i}(s, t, m, n)(\tilde{w}(s, t))^{\frac{r_{i}}{p}},=1,2, \ldots, l_{1}$,

$$
\begin{aligned}
& \tilde{d}_{i}(s, t, m, n)=d_{i}(s, t, m, n)(\tilde{w}(s, t))^{\frac{h_{i}}{p}}, \tilde{e}_{i}(s, t, m, n) \\
& \quad=e_{i}(s, t, m, n)(\tilde{w}(s, t))^{\frac{j_{i}}{p}}, i=1,2, \ldots, l_{2}, \\
& \tilde{w}(m, n)=\prod_{s=m+1}^{\infty}[1+w(s, n)], \\
& \tilde{\mu}=\sum_{i=1}^{l_{2}} \sum_{s=M+1}^{\infty} \sum_{t=N+1}^{\infty}\left\{\tilde{d}_{i}(s, t, M, N) \frac{h_{i}}{p} K_{3}^{\frac{h_{i}-p}{p}} \tilde{C}(s, t)\right. \\
& \left.\quad+\sum_{\xi=s}^{\infty} \sum_{\eta=t}^{\infty} \tilde{e}_{i}(\xi, \eta, M, N) \frac{j_{i}}{p} K_{4}^{\frac{j_{i}-p}{p}} \tilde{C}(\xi, \eta)\right\}, \\
& \tilde{C}(m, n)=\exp \left\{\sum_{s=m+1}^{\infty} \sum_{t=n+1}^{\infty} \tilde{B}(s, t, m, n)\right\}, \\
& \tilde{B}(s, t, m, n)=\sum_{i=1}^{l_{1}}\left[\tilde{b}_{i}(s, t, m, n) \frac{q_{i}}{p} K_{1}^{\frac{q_{i}-p}{p}}+\sum_{\xi=m_{0}}^{s} \sum_{\eta=n_{0}}^{t} \tilde{c}_{i}(\xi, \eta, m, n) \frac{r_{i}}{p} K_{2}^{\frac{r_{i}-p}{p}}\right] .
\end{aligned}
$$

Proof. Denote the right side of $(28)$ be $F(m, n)+\sum_{s=m+1}^{\infty} w(s, n) u^{p}(m, n)$. Then, we have

$$
u^{p}(m, n) \leq F(m, n)+\sum_{s=m+1}^{\infty} w(s, n) u^{p}(m, n) .
$$


Obviously $F(m, n)$ is nonincreasing in the first variable. So, by Lemma 2.1, we obtain

$$
u^{p}(m, n) \leq F(m, n) \sum_{s=m+1}^{\infty}[1+w(s, n)]=F(m, n) \tilde{w}(m, n),
$$

where $\tilde{w}(m, n)=\prod_{s=m+1}^{\infty}[1+w(s, n)]$. Define $F(m, n)=a(m, n)+v(m, n)$. Then

$$
u(m, n) \leq[(a(m, n)+v(m, n)) \tilde{w}(m, n)]^{\frac{1}{p}} .
$$

Furthermore, by (38) and Lemma 2.4, we have

$$
\begin{aligned}
& v(m, n) \leq \sum_{i=1}^{l_{1}} \sum_{s=m+1}^{\infty} \sum_{t=n+1}^{\infty}\left\{b_{i}(s, t, m, n)[(a(s, t)+v(s, t)) \tilde{w}(s, t)]^{\frac{q_{i}}{p}}\right. \\
& \left.+\sum_{\xi=s}^{\infty} \sum_{\eta=t}^{\infty} c_{i}(\xi, \eta, m, n)[(a(\xi, \eta)+v(\xi, \eta)) \tilde{w}(\xi, \eta)]^{\frac{r_{\dot{m}}}{p}}\right\} \\
& +\sum_{i=1}^{l_{2}} \sum_{s=M+1}^{\infty} \sum_{t=N+1}^{\infty}\left\{d_{i}(s, t, m, n)[(a(s, t)+v(s, t)) \tilde{w}(s, t)]^{\frac{h_{i}}{p}}\right. \\
& \left.+\sum_{\xi=s}^{\infty} \sum_{\eta=t}^{\infty} e_{i}(\xi, \eta, m, n)[(a(\xi, \eta)+v(\xi, \eta)) \tilde{w}(\xi, \eta)]^{\frac{j_{i}}{p}}\right\} \\
& \leq \sum_{i=1}^{l_{1}} \sum_{s=m+1}^{\infty} \sum_{t=n+1}^{\infty}\left\{b_{i}(s, t, m, n)(\tilde{w}(s, t))^{\frac{q_{i}}{p}}\left[\frac{q_{i}}{p} K_{1}^{\frac{q_{i}-p}{p}}(a(s, t)+v(s, t))+\frac{p-q_{i}}{p} K_{1}^{\frac{q_{i}}{p}}\right]\right. \\
& \left.+\sum_{\xi=s}^{\infty} \sum_{\eta=t}^{\infty} c_{i}(\xi, \eta, m, n)(\tilde{w}(\xi, \eta))^{\frac{r_{i}}{p}}\left[\frac{r_{i}}{p} K_{2}^{\frac{r_{i}-p}{p}}(a(\xi, \eta)+v(\xi, \eta))+\frac{p-r_{i}}{p} K_{2}^{\frac{r_{i}}{p}}\right]\right\} \\
& +\sum_{i=1}^{l_{2}} \sum_{s=M+1}^{\infty} \sum_{t=N+1}^{\infty}\left\{d_{i}(s, t, m, n)(\tilde{w}(s, t))^{\frac{h_{i}}{p}}\left[\frac{h_{i}}{p} K_{3}^{\frac{q_{i}-p}{p}}(a(s, t)+v(s, t))+\frac{p-h_{i}}{p} K_{3}^{\frac{h_{i}}{p}}\right]\right. \\
& \left.+\sum_{\xi=s}^{\infty} \sum_{\eta=t}^{\infty} e_{i}(\xi, \eta, m, n)(\tilde{w}(\xi, \eta))^{\frac{j_{i}}{p}}\left[\frac{j_{i}}{p} K_{4}^{\frac{j_{i}-p}{p}}(a(\xi, \eta)+v(\xi, \eta))+\frac{p-j_{i}}{p} K_{4}^{\frac{j_{i}}{p}}\right]\right\} \\
& =\sum_{i=1}^{l_{1}} \sum_{s=m+1}^{\infty} \sum_{t=n+1}^{\infty}\left\{\tilde{b}_{i}(s, t, m, n)\left[\frac{q_{i}}{p} K_{1}^{\frac{q_{i}-p}{p}}(a(s, t)+v(s, t))+\frac{p-q_{i}}{p} K_{1}^{\frac{q_{i}}{p}}\right]\right. \\
& \left.+\sum_{\xi=s}^{\infty} \sum_{\eta=t}^{\infty} \tilde{c}_{i}(\xi, \eta, m, n)\left[\frac{r_{i}}{p} K_{2}^{\frac{r_{i}-p}{p}}(a(\xi, \eta)+v(\xi, \eta))+\frac{p-r_{i}}{p} K_{2}^{\frac{r_{i}}{p}}\right]\right\} \\
& +\sum_{i=1}^{l_{2}} \sum_{s=M+1}^{\infty} \sum_{t=N+1}^{\infty}\left\{\tilde{d}_{i}(s, t, m, n)\left[\frac{h_{i}}{p} K_{3}^{\frac{q_{i}-p}{p}}(a(s, t)+v(s, t))+\frac{p-h_{i}}{p} K_{3}^{\frac{h_{i}}{p}}\right]\right. \\
& \left.+\sum_{\xi=s}^{\infty} \sum_{\eta=t}^{\infty} \tilde{e}_{i}(\xi, \eta, m, n)\left[\frac{j_{i}}{p} K_{4}^{\frac{j_{i}-p}{p}}(a(\xi, \eta)+v(\xi, \eta))+\frac{p-j_{i}}{p} K_{4}^{\frac{j_{i}}{p}}\right]\right\} \\
& =\tilde{H}(m, n)+\sum_{i=1}^{l_{1}} \sum_{s=m+1}^{\infty} \sum_{t=n+1}^{\infty}\left[\tilde{b}_{i}(s, t, m, n) \frac{q_{i}}{p} K_{1}^{\frac{q_{i}-p}{p}} v(s, t)\right. \\
& \left.+\sum_{\xi=s}^{\infty} \sum_{\eta=t}^{\infty} \tilde{c}_{i}(\xi, \eta, m, n) \frac{r_{i}}{p} K_{2}^{\frac{r_{i}-p}{p}} v(\xi, \eta)\right],
\end{aligned}
$$

where $\quad \tilde{H}(m, n)=\tilde{J}(m, n)+\sum_{i=1}^{l_{2}} \sum_{s=M+1}^{\infty} \sum_{t=N+1}^{\infty}\left\{\tilde{d}_{i}(s, t, m, n) \frac{h_{i}}{p} K_{3}^{\frac{h_{i}-p}{p}} v(s, t)+\sum_{\xi=s}^{\infty} \sum_{\eta=t}^{\infty} \tilde{e}_{i}(\xi, \eta, m, n) \frac{j_{i}}{p} K_{4}^{\frac{j_{i}-p}{p}} v(\xi, \eta)\right\}$, and $\tilde{J}(m, n), \tilde{b}_{i}, \tilde{c}_{i}, \tilde{d}_{i}, \tilde{e}_{i}$ are defined in (30)-(32), respectively. Then, using $\tilde{H}(m, n)$ is nonincreasing in every variable, we obtain 


$$
\begin{aligned}
v(m, n) \leq & \tilde{H}(M, N)+\sum_{i=1}^{l_{1}} \sum_{s=m+1}^{\infty} \sum_{t=n+1}^{\infty}\left[\tilde{b}_{i}(s, t, m, n) \frac{q_{i}}{p} K_{1}^{\frac{q_{i}-p}{p}} v(s, t)\right. \\
& \left.+\sum_{\xi=s}^{\infty} \sum_{\eta=t}^{\infty} \tilde{c}_{i}(\xi, \eta, m, n) \frac{r_{i}}{p} K_{2}^{\frac{r_{i}-p}{p}} v(\xi, \eta)\right] \\
\leq & \tilde{H}(M, N)+\sum_{i=1}^{l_{1}} \sum_{s=m+1}^{\infty} \sum_{t=n+1}^{\infty}\left[\tilde{b}_{i}(s, t, m, n) \frac{q_{i}}{p} K_{1}^{\frac{q_{i}-p}{p}}\right. \\
& \left.+\sum_{\xi=s}^{\infty} \sum_{\eta=t}^{\infty} \tilde{c}_{i}(\xi, \eta, m, n) \frac{r_{i}}{p} K_{2}^{\frac{r_{i}-p}{p}}\right] v(s, t) . \\
= & \tilde{H}(M, N)+\sum_{s=m+1}^{\infty} \sum_{t=n+1}^{\infty} \tilde{B}(s, t, m, n) v(s, t),
\end{aligned}
$$

where $B(s, t, m, n)$ is defined in (36). Using $B(s, t, m, n)$ is nonincreasing in the last two variables, by a suitable application of Corollary 2.3, we obtain

$$
v(m, n) \leq \tilde{H}(M, N) \exp \left\{\sum_{s=m+1}^{\infty} \sum_{t=n+1}^{\infty} \tilde{B}(s, t, m, n)\right\}=\tilde{H}(M, N) \tilde{C}(m, n),
$$

where $\tilde{C}(m, n)$ is defined in (35). Furthermore, considering the definition of $\tilde{H}(m, n)$ and (40), we have

$$
\begin{aligned}
\tilde{H}(M, N)= & \tilde{J}(M, N)+\sum_{i=1}^{l_{2}} \sum_{s=M+1}^{\infty} \sum_{t=N+1}^{\infty}\left\{\tilde{d}_{i}(s, t, M, N) \frac{h_{i}}{p} K_{3}^{\frac{h_{i}-p}{p}} v(s, t)\right. \\
& \left.+\sum_{\xi=s}^{\infty} \sum_{\eta=t}^{\infty} \tilde{e}_{i}(\xi, \eta, M, N) \frac{j_{i}}{p} K_{4}^{\frac{j_{i}-p}{p}} v(\xi, \eta)\right\} \\
\leq & \tilde{J}(M, N)+\sum_{i=1}^{l_{2}} \sum_{s=M+1}^{\infty} \sum_{t=N+1}^{\infty}\left\{\tilde{d}_{i}(s, t, M, N) \frac{h_{i}}{p} K_{3}^{\frac{h_{i}-p}{p}} \tilde{H}(M, N) \tilde{C}(s, t)\right. \\
& \left.+\sum_{\xi=s}^{\infty} \sum_{\eta=t}^{\infty} \tilde{e}_{i}\left(\xi, \eta, m_{1}, n_{1}\right) \frac{j_{i}}{p} K_{4}^{\frac{j_{i}-p}{p}} \tilde{H}(M, N) \tilde{C}(\xi, \eta)\right\} \\
= & \tilde{J}(M, N)+\tilde{H}(M, N) \sum_{i=1}^{l_{2}} \sum_{s=M+1}^{\infty} \sum_{t=N+1}^{\infty}\left\{\tilde{d}_{i}(s, t, M, N) \frac{h_{i}}{p} K_{3}^{\frac{h_{i}-p}{v}} \tilde{C}(s, t)\right. \\
& \left.+\sum_{\xi=s}^{\infty} \sum_{\eta=t}^{\infty} \tilde{e}_{i}(\xi, \eta, M, N) \frac{j_{i}}{p} K_{4}^{\frac{j_{i}-p}{p}} C(\xi, \eta)\right\}, \\
= & \tilde{J}(M, N)+\tilde{H}(M, N) \tilde{\mu},
\end{aligned}
$$

where $\tilde{\mu}$ is defined in (34). Then, according to $\tilde{\mu}<1$, we have

$$
\tilde{H}(M, N) \leq \frac{\tilde{J}(M, N)}{1-\tilde{\mu}} .
$$

From (40) and (41), we deduce

$$
v(m, n) \leq \frac{\tilde{J}(M, N)}{1-\tilde{\mu}} \tilde{C}(m, n) .
$$


Then, combining (38) and (42), we obtain the desired result.

Remark 2. As one can see, the established results above mainly deal with VolterraFredholm type discrete inequalities with four iterated sums and infinite sum upper limit, and they are different from the results presented in [3]. Furthermore, without considering the slight difference in infinite sum upper limit, Theorem 2.6 is generalization of [[3], Theorem 2.5]. If we take $w(m, n) \equiv 0, b_{i}(s, t, m, n)=b_{i}(s, t), c_{i}(\xi, \eta, m, n)$ $=0, i=1,2, \ldots, l_{1}, d_{i}(s, t, m, n)=d_{i}(s, t), e_{i}(\xi, \eta, m, n) \equiv 0, i=1,2, \ldots, l_{2}$, then Theorem 2.6 reduces to [[3], Theorem 2.5$]$.

\section{Analysis of the properties of the solutions of Eq. (1)}

In this section, we will present some results on the boundedness, uniqueness, and continuous dependence on initial data of the solutions of the Volterra-Fredholm type difference equation shown in (1).

Theorem 3.1. In Eq. (1), suppose $g_{1}(m, n) \equiv 0,\left|F_{1 i}(s, t, m, n, z)\right| \leq b_{i}(s, t, m, n) \phi(\mid$ $z \mid),\left|F_{2 i}(s, t, m, n, z)\right| \leq c_{i}(s, t, m, n) \phi(|z|), i=1,2, \ldots, l_{1},\left|G_{1 i}(s, t, m, n, z)\right| \leq d_{i}(s, t, m$, $n)|z|^{p},\left|G_{2 i}(s, t, m, n, z)\right| \leq e_{i}(s, t, m, n)|z|^{p}, i=1,2, \ldots, l_{2}$, where $b_{i}, c_{i}, d_{i}, e_{i}, \phi$ are defined as in Theorem 2.5 , then we have the following estimate

$$
|z(m, n)| \leq\left\{G^{-1}\left[G\left(J^{-1}(C(M, N))\right)+C(m, n)\right] \bar{g}_{2}(m, n)\right\}^{\frac{1}{p}},
$$

provided that $0<\mu<1$, where $G, J$ are defined as in Theorem 2.5, and

$$
\begin{aligned}
\bar{g}_{2}(m, n) & =\prod_{s=m+1}^{\infty}\left[1+\left|g_{2}(s, n)\right|\right], \\
C(m, n) & =\sum_{s=m+1}^{\infty} \sum_{t=n+1}^{\infty} B(s, t, m, n), \\
B(s, t, m, n) & =\sum_{i=1}^{l_{1}}\left[b_{i}(s, t, m, n) \varphi\left(\bar{g}_{2}^{\frac{1}{p}}(s, t)\right)+\sum_{\xi=s}^{\infty} \sum_{\eta=t}^{\infty} c_{i}(\xi, \eta, m, n) \varphi\left(\bar{g}_{2}^{\frac{1}{p}}(\xi, \eta)\right)\right], \\
\mu & =\sum_{i=1}^{l_{2}} \sum_{s=M+1}^{\infty} \sum_{t=N+1}^{\infty}\left[d_{i}(s, t, M, N) \bar{g}_{2}(s, t)+\sum_{\xi=s}^{\infty} \sum_{\eta=t}^{\infty} e_{i}(\xi, \eta, M, N) \bar{g}_{2}(\xi, \eta)\right] .
\end{aligned}
$$

Proof. Considering $g_{1}(m, n) \equiv 0$, from (1) we deduce

$$
\begin{aligned}
|z(m, n)|^{p} \leq & \sum_{s=m+1}^{\infty}\left|g_{2}(s, n)\right||z(s, n)|^{p}+\sum_{i=1}^{l_{1}} \sum_{s=m+1}^{\infty} \sum_{t=n+1}^{\infty}\left[\left|F_{1 i}(s, t, m, n, z(s, t))\right|\right. \\
& \left.+\sum_{\xi=s}^{\infty} \sum_{\eta=t}^{\infty}\left|F_{2 i}(\xi, \eta, m, n, z(\xi, \eta))\right|\right] \\
& +\sum_{i=1}^{l_{2}} \sum_{s=M+1}^{\infty} \sum_{t=N+1}^{\infty}\left[\left|G_{1 i}(s, t, m, n, z(s, t))\right|+\sum_{\xi=s}^{\infty} \sum_{\eta=t}^{\infty}\left|G_{2 i}(\xi, \eta, m, n, z(\xi, \eta))\right|\right] \\
\leq & \sum_{s=m+1}^{\infty}\left|g_{2}(s, n)\right||z(s, n)|^{p}+\sum_{i=1}^{l_{1}} \sum_{s=m+1}^{\infty} \sum_{t=n+1}^{\infty}\left[b_{i}(s, t, m, n) \varphi(|z(s, t)|)\right. \\
& \left.+\sum_{\xi=s}^{\infty} \sum_{\eta=t}^{\infty} c_{i}(s, t, m, n) \varphi(|z(\xi, \eta)|)\right] \\
& +\sum_{i=1}^{l_{2}} \sum_{s=M+1}^{\infty} \sum_{t=N+1}^{\infty}\left[d_{i}(s, t, m, n)|z(s, t)|^{p}+\sum_{\xi=s}^{\infty} \sum_{\eta=t}^{\infty} e_{i}(s, t, m, n)|z(\xi, \eta)|^{p}\right] .
\end{aligned}
$$


Then, a suitable application of Theorem 2.5 yields the desired result.

Theorem 3.2. Suppose $\quad\left|F_{1 i}(s, t, m, n, z)\right| \leq b_{i}(s, t, m, n)|z|^{q_{i}}$, $\left|F_{2 i}(s, t, m, n, z)\right| \leq c_{i}(s, t, m, n)|z|^{r_{i}}, \quad i=1,2, \ldots, l_{1},\left|G_{1 i}(s, t, m, n, z)\right| \leq d_{i}(s, t, m, n)|z|^{h_{i}}$, $\left|G_{2 i}(s, t, m, n, z)\right| \leq e_{i}(s, t, m, n)|z|^{j_{i}}, i=1,2, \ldots, l_{2}$, where $b_{i}, c_{i}, d_{i}, e_{i}, q_{i}, r_{i}, h_{i}, j_{i}$ are defined as in Theorem 2.6, then we have the following estimate

$$
|z(m, n)| \leq\left\{\left[\left|g_{1}(m, n)\right|+\frac{\tilde{J}(M, N)}{1-\tilde{\mu}} \tilde{C}(m, n)\right] \tilde{g}_{2}(m, n)\right\}^{\frac{1}{P}},
$$

provided that $\tilde{\mu}<1$, where

$$
\begin{aligned}
& \tilde{J}(m, n)=\sum_{i=1}^{l_{1}} \sum_{s=m+1}^{\infty} \sum_{t=n+1}^{\infty}\left\{\tilde{b}_{i}(s, t, m, n)\left[\frac{q_{i}}{p} K_{1}^{\frac{q_{i}-p}{p}}\left|g_{1}(s, t)\right|+\frac{p-q_{i}}{p} K_{1}^{\frac{q_{i}}{p}}\right]\right. \\
& \left.+\sum_{\xi=s}^{\infty} \sum_{\eta=t}^{\infty} \tilde{c}_{i}(\xi, \eta, m, n)\left[\frac{r_{i}}{p} K_{2}^{\frac{r_{i}-p}{p}}\left|g_{1}(\xi, \eta)\right|+\frac{p-r_{i}}{p} K_{2}^{\frac{r_{i}}{p}}\right]\right\} \\
& +\sum_{i=1}^{l_{2}} \sum_{s=M+1}^{\infty} \sum_{t=N+1}^{\infty}\left\{\tilde{d}_{i}(s, t, m, n)\left[\frac{h_{i}}{p} K_{3}^{\frac{h_{i}-p}{p}}\left|g_{1}(s, t)\right|+\frac{p-h_{i}}{p} K_{3}^{\frac{h_{i}}{p}}\right]\right. \\
& \left.+\sum_{\xi=s}^{\infty} \sum_{\eta=t}^{\infty} \tilde{e}_{i}(\xi, \eta, m, n)\left[\frac{j_{i}}{p} K_{4}^{\frac{j_{i}-p}{p}}\left|g_{1}(\xi, \eta)\right|+\frac{p-j_{i}}{p} K_{4}^{\frac{j_{j}}{p}}\right]\right\} \text {, } \\
& \tilde{b}_{i}(s, t, m, n)=b_{i}(s, t, m, n)\left(\tilde{g}_{2}(s, t)\right)^{\frac{q_{i}}{p}}, \tilde{c}_{i}(s, t, m, n)=c_{i}(s, t, m, n)\left(\tilde{g}_{2}(s, t)\right)^{\frac{r_{i}}{p}}, i=1,2, \ldots, l_{1} \text {, } \\
& \tilde{d}_{i}(s, t, m, n)=d_{i}(s, t, m, n)\left(\tilde{g}_{2}(s, t)\right)^{\frac{h_{i}}{p}}, \tilde{e}_{i}(s, t, m, n)=e_{i}(s, t, m, n)\left(\tilde{g}_{2}(s, t)\right)^{\frac{j_{i}}{p}}, i=1,2, \ldots, l_{2}, \\
& \tilde{g}_{2}(m, n)=\prod_{s=m+1}^{\infty}\left[1+\left|g_{2}(s, n)\right|\right], \\
& \tilde{\mu}=\sum_{i=1}^{l_{2}} \sum_{s=M+1}^{\infty} \sum_{t=N+1}^{\infty}\left\{\tilde{d}_{i}(s, t, M, N) \frac{h_{i}}{p} K_{3}^{\frac{h_{i}-p}{p}} \tilde{C}(s, t)+\sum_{\xi=s}^{\infty} \sum_{\eta=t}^{\infty} \tilde{e}_{i}(\xi, \eta, M, N) \frac{j_{i}}{p} K_{4}^{\frac{j_{i}-p}{p}} \tilde{C}(\xi, \eta)\right\}, \\
& \tilde{C}(m, n)=\exp \left\{\sum_{s=m+1}^{\infty} \sum_{t=n+1}^{\infty} \tilde{B}(s, t, m, n)\right\} \text {, } \\
& \tilde{B}(s, t, m, n)=\sum_{i=1}^{l_{1}}\left[\tilde{b}_{i}(s, t, m, n) \frac{q_{i}}{p} K_{1}^{\frac{q_{i}-p}{p}}+\sum_{\xi=m_{0}}^{s} \sum_{\eta=n_{0}}^{t} \tilde{c}_{i}(\xi, \eta, m, n) \frac{r_{i}}{p} K_{2}^{\frac{r_{i}-p}{p}}\right] .
\end{aligned}
$$

Proof. From (1), we deduce

$$
\begin{aligned}
|z(m, n)|^{p} \leq & \left|g_{1}(m, n)\right|+\sum_{s=m+1}^{\infty}\left|g_{2}(s, n)\right||z(s, n)|^{p} \\
& +\sum_{i=1}^{l_{1}} \sum_{s=m+1}^{\infty} \sum_{t=n+1}^{\infty}\left[\left|F_{1 i}(s, t, m, n, z(s, t))\right|+\sum_{\xi=s}^{\infty} \sum_{\eta=t}^{\infty}\left|F_{2 i}(\xi, \eta, m, n, z(\xi, \eta))\right|\right] \\
& +\sum_{i=1}^{l_{2}} \sum_{s=M+1}^{\infty} \sum_{t=N+1}^{\infty}\left[\left|G_{1 i}(s, t, m, n, z(s, t))\right|+\sum_{\xi=s}^{\infty} \sum_{\eta=t}^{\infty}\left|G_{2 i}(\xi, \eta, m, n, z(\xi, \eta))\right|\right] \\
\leq & \left|g_{1}(m, n)\right|+\sum_{s=m+1}^{\infty}\left|g_{2}(s, n)\right||z(s, n)|^{p} \\
& +\sum_{i=1}^{l_{1}} \sum_{s=m+1}^{\infty} \sum_{t=n+1}^{\infty}\left[b_{i}\left(s, t, m, n|z(s, t)|^{q_{i}}+\sum_{\xi=s}^{\infty} \sum_{\eta=t}^{\infty} c_{i}(s, t, m, n)|z(\xi, \eta)|^{r_{i}}\right]\right. \\
& +\sum_{i=1}^{l_{2}} \sum_{s=M+1}^{\infty} \sum_{t=N+1}^{\infty}\left[d_{i}(s, t, m, n)|z(s, t)|^{h_{i}}+\sum_{\xi=s}^{\infty} \sum_{\eta=t}^{\infty} e_{i}(s, t, m, n)|z(\xi, \eta)|^{j_{i}}\right] .
\end{aligned}
$$

Then, a suitable application of Theorem 2.6 yields the desired result. 
The following theorem deals with the uniqueness of the solutions of Eq. (1).

Theorem 3.3. Suppose, $\left|F_{1 i}(s, t, m, n, u)-F_{1 i}(s, t, m, n, v)\right| \leq b_{i}(s, t, m, n)\left|u^{p}-v^{p}\right|, \mid$ $F_{2 i}(s, t, m, n, u)-F_{2 i}(s, t, m, n, v)\left|\leq c_{i}(s, t, m, n)\right| u^{p}-v^{p}\left|, i=1,2, \ldots, l_{1},\right| G_{1 i}(s, t, m, n$, $u)-G_{1 i}(s, t, m, n, v)\left|\leq d_{i}(s, t, m, n)\right| u^{p}-v^{p}|,| G_{2 i}(s, t, m, n, u)-G_{2 i}(s, t, m, n, v) \mid \leq e_{i}$ $(s, t, m, n)\left|u^{p}-v^{p}\right|, i=1,2, \ldots, l_{2}$ hold for $\forall u, v \in \mathbb{R}$, where $b_{i}, c_{i}, d_{i}, e_{i}$ are defined as in Theorem 2.6 , and $\tilde{\mu}=\sum_{i=1}^{l_{2}} \sum_{s=M+1}^{\infty} \sum_{t=N+1}^{\infty}\left\{\tilde{d}_{i}(s, t, M, N) \tilde{C}(s, t)+\sum_{\xi=m_{0}}^{s} \sum_{\eta=n_{0}}^{t} \tilde{e}_{i}(\xi, \eta, M, N) \tilde{C}(\xi, \eta)\right\}<1$, where

$$
\left\{\begin{array}{l}
\tilde{d}_{i}(s, t, m, n)=d_{i}(s, t, m, n) \tilde{g}_{2}(s, t), \tilde{e}_{i}(s, t, m, n)=e_{i}(s, t, m, n) \tilde{g}_{2}(s, t), i=1,2, \ldots, l_{2}, \\
\tilde{g}_{2}(m, n)=\prod_{s=m+1}^{\infty}\left[1+\left|g_{2}(s, n)\right|\right] \\
\tilde{C}(m, n)=\exp \left\{\sum_{s=m+1}^{\infty} \sum_{t=n+1}^{\infty} \tilde{B}(s, t, m, n)\right\} \\
\tilde{B}(s, t, m, n)=\sum_{i=1}^{l_{1}}\left[\tilde{b}_{i}(s, t, m, n)+\sum_{\xi=s}^{\infty} \sum_{\eta=t}^{\infty} \tilde{c}_{i}(\xi, \eta, m, n)\right] \\
\tilde{b}_{i}(s, t, m, n)=b_{i}(s, t, m, n) \tilde{g}_{2}(s, t), \tilde{c}_{i}(s, t, m, n)=c_{i}(s, t, m, n) \tilde{g}_{2}(s, t), i=1,2, \ldots, l_{1},
\end{array}\right.
$$

then Eq. (1) has at most one solution.

Proof. Suppose, $z_{1}(m, n), z_{2}(m, n)$ are two solutions of Eq. (1). Then

$$
\begin{aligned}
\left|z_{1}^{p}(m, n)-z_{2}^{p}(m, n)\right| \leq & \sum_{s=m+1}^{\infty}\left|g_{2}(s, n)\right|\left|z_{1}^{p}(s, n)-z_{2}^{p}(s, n)\right| \\
& +\sum_{i=1}^{l_{1}} \sum_{s=m+1}^{\infty} \sum_{t=n+1}^{\infty}\left[\left|F_{1 i}\left(s, t, m, n, z_{1}(s, t)\right)-F_{1 i}\left(s, t, m, n, z_{2}(s, t)\right)\right|\right. \\
& \left.+\sum_{\xi=s}^{\infty} \sum_{\eta=t}^{\infty}\left|F_{2 i}\left(\xi, \eta, m, n, z_{1}(\xi, \eta)\right)-F_{2 i}\left(\xi, \eta, m, n, z_{2}(\xi, \eta)\right)\right|\right] \\
& +\sum_{i=1}^{l_{2}} \sum_{s=M+1}^{\infty} \sum_{t=N+1}^{\infty}\left[\left|G_{1 i}\left(s, t, m, n, z_{1}(s, t)\right)-G_{1 i}\left(s, t, m, n, z_{2}(s, t)\right)\right|\right. \\
& \left.+\sum_{\xi=s}^{\infty} \sum_{\eta=t}^{\infty}\left|G_{2 i}\left(\xi, \eta, m, n, z_{1}(\xi, \eta)\right)-G_{2 i}\left(\xi, \eta, m, n, z_{2}(\xi, \eta)\right)\right|\right] \\
\leq & \sum_{s=m+1}^{\infty}\left|g_{2}(s, n)\right|\left|z_{1}^{p}(s, n)-z_{2}^{p}(s, n)\right| \\
& +\sum_{\mathbf{i}=\mathbf{1}}^{\mathbf{l}_{1}} \sum_{\mathbf{s}=\mathbf{m}+\mathbf{1}}^{\infty} \sum_{\mathbf{t}=\mathbf{n}+\mathbf{1}}^{\infty}\left[\mathbf{b}_{\mathbf{i}}(\mathbf{s}, \mathbf{t}, \mathbf{m}, \mathbf{n})\left|\mathbf{z}_{1}^{\mathbf{p}}(\mathbf{s}, \mathbf{t})-\mathbf{z}_{2}^{\mathrm{p}}(\mathbf{s}, \mathbf{t})\right|\right. \\
& \left.+\sum_{\xi=\mathbf{s}}^{\infty} \sum_{\eta=\mathbf{t}}^{\infty} \mathbf{c}_{\mathbf{i}}(\xi, \eta, \mathbf{m}, \mathbf{n})\left|\mathbf{z}_{1}^{\mathbf{p}}(\xi, \eta)-\mathbf{z}_{2}^{\mathbf{p}}(\xi, \eta)\right|\right] \\
& +\sum_{\mathbf{i}=\mathbf{1}}^{\mathrm{l}_{2}} \sum_{\mathbf{s}=\mathbf{m}+\mathbf{1}}^{\infty} \sum_{\mathbf{t}=\mathbf{n}+\mathbf{1}}^{\infty}\left[\mathbf{d}_{\mathbf{i}}(\mathbf{s}, \mathbf{t}, \mathbf{m}, \mathbf{n})\left|\mathbf{z}_{\mathbf{1}}^{\mathbf{p}}(\mathbf{s}, \mathbf{t})-\mathbf{z}_{2}^{\mathrm{p}}(\mathbf{s}, \mathbf{t})\right|\right. \\
& \left.+\sum_{\xi=\mathbf{s}}^{\infty} \sum_{\eta=\mathbf{t}}^{\infty} \mathbf{e}_{\mathbf{i}}(\xi, \eta, \mathbf{m}, \mathbf{n})\left|\mathbf{z}_{1}^{\mathbf{p}}(\xi, \eta)-\mathbf{z}_{2}^{\mathbf{p}}(\xi, \eta)\right|\right] .
\end{aligned}
$$

Treat $\left|z_{1}^{p}(m, n)-z_{2}^{p}(m, n)\right|$ as one variable, and a suitable application of Theorem 2.6 yields $\left|z_{1}^{p}(m, n)-z_{2}^{p}(m, n)\right| \leq 0$, which implies $z_{1}^{p}(m, n) \equiv z_{2}^{p}(m, n)$. Since $p$ is an odd number, then we have $z_{1}(m, n) \equiv z_{2}(m, n)$, and the proof is complete. 
Finally, we study the continuous dependence of the solutions of Eq. (1) on the functions $g_{1}, F_{1 i}, F_{2 i}, G_{1 i}, G_{2 i}$.

Theorem 3:4. Suppose, $g_{2}(m, n) \equiv 0, z(m, n)$ is a solution of Eq. (1), $\mid F_{1 i}(s, t, m, n, u)$ - $F_{1 i}(s, t, m, n, v)\left|\leq b_{i}(s, t, m, n)\right| u^{p}-v^{p}|,| F_{2 i}(s, t, m, n, u)-F_{2 i}(s, t, m, n, v) \mid \leq c_{i}(s, t$, $m, n)\left|u^{p}-v^{p}\right|, i=1,2, \ldots, l_{1},\left|G_{1 i}(s, t, m, n, u)-G_{1 i}(s, t, m, n, v)\right| \leq d_{i}(s, t, m, n) \mid u^{p}-$ $v^{p}|,| G_{2 i}(s, t, m, n, u)-G_{2 i}(s, t, m, n, v)\left|\leq e_{i}(s, t, m, n)\right| u^{p}-v^{p} \mid, i=1,2, \ldots, l_{2}$ hold for $\forall u, v \in \mathbb{R}$, where $b_{i}, c_{i}, d_{i}, e_{i}$ are defined as in Theorem 2.6, and furthermore, $\left.\left.\left|g_{1}(m, n)-\bar{g}_{1}(m, n)\right|+\sum_{i=1}^{l_{1}} \sum_{s=n+1}^{\infty} \sum_{i=n+1}^{\infty}\left[\left|F_{1 i}(s, t, m, n, \bar{z}(s, t))-\bar{F}_{1 i}(s, t, m, n, \bar{z}(s, t))\right|+\sum_{\xi=s}^{\infty} \sum_{n=t}^{\infty} \mid F_{2 i}(\xi, \eta, m, m, n, \bar{z}(\xi, \eta))\right)-\bar{F}_{2 i}(\xi, \eta, m, n, n, \bar{z}(\xi, \eta))\right)\right]$ $\left.+\sum_{i=1}^{l_{2}} \sum_{1=M+1}^{\infty} \sum_{t=N+1}^{\infty}\left[\left|G_{1 i}(s, t, m, n, \bar{z}(s, t))-\bar{G}_{1 i}(s, t, m, n, \bar{z}(s, t))\right|+\sum_{\xi=s}^{\infty} \sum_{n=t}^{\infty} \mid G_{2 i}(\xi, \eta, m, n, n, \bar{z}(\xi, \eta))-\bar{G}_{2 i}(\xi, \eta, m, m, n, \bar{z}(\xi, \eta))\right)\right] \leq \varepsilon$ , where $\varepsilon$ $>0$ is a constant, and $\bar{z}(m, n) \in \wp(\Omega)$ is the solution of the following difference equation

$$
\begin{aligned}
\bar{z}^{p}(m, n)= & \bar{g}_{1}(m, n)+\sum_{i=1}^{l_{1}} \sum_{s=m+1}^{\infty} \sum_{t=n+1}^{\infty}\left[\bar{F}_{1 i}(s, t, m, n, \bar{z}(s, t))+\sum_{\xi=s}^{\infty} \sum_{\eta=t}^{\infty} \bar{F}_{2 i}(\xi, \eta, m, n, \bar{z}(\xi, \eta))\right] \\
& +\sum_{i=1}^{l_{2}} \sum_{s=M+1}^{\infty} \sum_{t=N+1}^{\infty}\left[\bar{G}_{1 i}(s, t, m, n, \bar{z}(s, t))+\sum_{\xi=s}^{\infty} \sum_{\eta=t}^{\infty} \bar{G}_{2 i}(\xi, \eta, m, n, \bar{z}(\xi, \eta))\right],
\end{aligned}
$$

where $\bar{g}_{1}(m, n) \in \wp(\Omega), \overline{\mathbf{F}}_{1 \mathbf{i}}, \overline{\mathbf{F}}_{2 \mathbf{i}} \in \wp\left(\Omega^{2} \times \mathbb{R}\right), \mathbf{i}=\mathbf{1}, \mathbf{2}, \ldots, \mathbf{l}_{\mathbf{1}}$ and $\overline{\mathbf{G}}_{1 \mathbf{i}}, \overline{\mathbf{G}}_{2 \mathbf{i}} \in \wp\left(\Omega^{2} \times \mathbb{R}\right)$, $\mathbf{i}=\mathbf{1}, 2, \ldots, \mathbf{l}_{2}$, then we have

$$
\left|z^{p}(m, n)-\bar{z}^{p}(m, n)\right| \leq \varepsilon\left\{\left[1+\frac{\tilde{J}(M, N)}{1-\tilde{\mu}} \tilde{C}(m, n)\right] \tilde{w}(m, n)\right\},
$$

provided that $\tilde{\mu}<1$, where

$$
\begin{aligned}
\tilde{J}(m, n)= & \sum_{i=1}^{l_{1}} \sum_{s=m+1}^{\infty} \sum_{t=n+1}^{\infty}\left\{b_{i}(s, t, m, n)+\sum_{\xi=s}^{\infty} \sum_{\eta=t}^{\infty} c_{i}(\xi, \eta, m, n)\right\} \\
& +\sum_{i=1}^{l_{2}} \sum_{s=M+1}^{\infty} \sum_{t=N+1}^{\infty}\left\{d_{i}(s, t, m, n)+\sum_{\xi=s}^{\infty} \sum_{\eta=t}^{\infty} e_{i}(\xi, \eta, m, n)\right\}, \\
\tilde{\mu}= & \sum_{i=1}^{l_{2}} \sum_{s=M+1}^{\infty} \sum_{t=N+1}^{\infty}\left\{d_{i}(s, t, M, N) \tilde{C}(s, t)+\sum_{\xi=s}^{\infty} \sum_{\eta=t}^{\infty} e_{i}(\xi, \eta, M, N) \tilde{C}(\xi, \eta)\right\}, \\
\tilde{C}(m, n)= & \exp \left\{\sum_{s=m+1}^{\infty} \sum_{t=n+1}^{\infty} \tilde{B}(s, t, m, n)\right\}, \\
\tilde{B}(s, t, m, n)= & \sum_{i=1}^{l_{1}}\left[b_{i}(s, t, m, n)+\sum_{\xi=m_{0}}^{s} \sum_{\eta=n_{0}}^{t} c_{i}(\xi, \eta, m, n)\right] .
\end{aligned}
$$


Proof. From (1) and (45), we deduce

$$
\begin{aligned}
& \left|z^{p}(m, n)-\bar{z}^{p}(m, n)\right| \leq\left|g_{1}(m, n)-\bar{g}_{1}(m, n)\right| \\
& +\sum_{i=1}^{l_{1}} \sum_{s=m+1}^{\infty} \sum_{t=n+1}^{\infty}\left[\left|F_{1 i}(s, t, m, n, z(s, t))-\bar{F}_{1 i}(s, t, m, n, \bar{z}(s, t))\right|\right. \\
& \left.+\sum_{\xi=s}^{\infty} \sum_{\eta=t}^{\infty}\left|F_{2 i}(\xi, \eta, m, n, z(\xi, \eta))-\bar{F}_{2 i}(\xi, \eta, m, n, \bar{z}(\xi, \eta))\right|\right] \\
& +\sum_{i=1}^{l_{2}} \sum_{s=M+1}^{\infty} \sum_{t=N+1}^{\infty}\left[\left|G_{1 i}(s, t, m, n, z(s, t))-\bar{G}_{1 i}(s, t, m, n, \bar{z}(s, t))\right|\right. \\
& \left.+\sum_{\xi=s}^{\infty} \sum_{\eta=t}^{\infty}\left|G_{2 i}(\xi, \eta, m, n, z(\xi, \eta))-\bar{G}_{2 i}(\xi, \eta, m, n, \bar{z}(\xi, \eta))\right|\right] \\
& \leq\left|g_{1}(m, n)-\bar{g}_{1}(m, n)\right| \\
& +\sum_{i=1}^{l_{1}} \sum_{s=m+1}^{\infty} \sum_{t=n+1}^{\infty}\left[\left|F_{1 i}(s, t, m, n, z(s, t))-F_{1 i}(s, t, m, n, \bar{z}(s, t))\right|\right. \\
& +\sum_{i=1}^{l_{1}} \sum_{s=m+1}^{\infty} \sum_{t=n+1}^{\infty}\left[\left|F_{1 i}(s, t, m, n, \bar{z}(s, t))-\bar{F}_{1 i}(s, t, m, n, \bar{z}(s, t))\right|\right. \\
& \left.+\sum_{\xi=s}^{\infty} \sum_{\eta=t}^{\infty}\left|F_{2 i}(\xi, \eta, m, n, z(\xi, \eta))-F_{2 i}(\xi, \eta, m, n, \bar{z}(\xi, \eta))\right|\right] \\
& \left.+\sum_{\xi=s}^{\infty} \sum_{\eta=t}^{\infty}\left|F_{2 i}(\xi, \eta, m, n, \bar{z}(\xi, \eta))-\bar{F}_{2 i}(\xi, \eta, m, n, \bar{z}(\xi, \eta))\right|\right] \\
& +\sum_{i=1}^{l_{2}} \sum_{s=M+1}^{\infty} \sum_{t=N+1}^{\infty}\left[\left|G_{1 i}(s, t, m, n, z(s, t))-G_{1 i}(s, t, m, n, \bar{z}(s, t))\right|\right. \\
& +\sum_{i=1}^{l_{2}} \sum_{s=M+1}^{\infty} \sum_{t=N+1}^{\infty}\left[\left|G_{1 i}(s, t, m, n, \bar{z}(s, t))-\bar{G}_{1 i}(s, t, m, n, \bar{z}(s, t))\right|\right. \\
& \left.+\sum_{\xi=s}^{\infty} \sum_{\eta=t}^{\infty}\left|G_{2 i}(\xi, \eta, m, n, z(\xi, \eta))-G_{2 i}(\xi, \eta, m, n, \bar{z}(\xi, \eta))\right|\right] \\
& \left.+\sum_{\xi=s}^{\infty} \sum_{\eta=t}^{\infty}\left|G_{2 i}(\xi, \eta, m, n, \bar{z}(\xi, \eta))-\bar{G}_{2 i}(\xi, \eta, m, n, \bar{z}(\xi, \eta))\right|\right] \\
& \leq \varepsilon+\sum_{i=1}^{l_{1}} \sum_{s=m+1}^{\infty} \sum_{t=n+1}^{\infty}\left[\left|F_{1 i}(s, t, m, n, z(s, t))-F_{1 i}(s, t, m, n, \bar{z}(s, t))\right|\right. \\
& \left.+\sum_{\xi=s}^{\infty} \sum_{\eta=t}^{\infty}\left|F_{2 i}(\xi, \eta, m, n, z(\xi, \eta))-F_{2 i}(\xi, \eta, m, n, \bar{z}(\xi, \eta))\right|\right] \\
& +\sum_{i=1}^{l_{2}} \sum_{s=M+1}^{\infty} \sum_{t=N+1}^{\infty}\left[\left|G_{1 i}(s, t, m, n, z(s, t))-G_{1 i}(s, t, m, n, \bar{z}(s, t))\right|\right. \\
& \left.+\sum_{\xi=s}^{\infty} \sum_{\eta=t}^{\infty}\left|G_{2 i}(\xi, \eta, m, n, z(\xi, \eta))-G_{2 i}(\xi, \eta, m, n, \bar{z}(\xi, \eta))\right|\right] \\
& \leq \varepsilon+\sum_{\mathbf{i}=1}^{\mathrm{l}_{1}} \sum_{\mathbf{s}=\mathbf{m}+1}^{\infty} \sum_{\mathbf{t}=\mathbf{n}+1}^{\infty}\left[\mathbf{b}_{\mathbf{i}}(\mathbf{s}, \mathbf{t}, \mathbf{m}, \mathbf{n})\left|\mathbf{z}^{\mathrm{P}}(\mathbf{s}, \mathbf{t})-\overline{\mathbf{z}}^{\mathrm{p}}(\mathbf{s}, \mathbf{t})\right|\right. \\
& \left.+\sum_{\xi=\mathbf{s}}^{\infty} \sum_{\eta=\mathbf{t}}^{\infty} \mathbf{c}_{\mathbf{i}}(\xi, \eta, \mathbf{m}, \mathbf{n}) \mid \mathbf{z}^{\mathbf{P}}(\xi, \eta)-\overline{\mathbf{z}}^{\mathbf{P}}(\xi, \eta)\right]
\end{aligned}
$$

Treat $\left|z^{p}(m, n)-\bar{z}^{p}(m, n)\right|$ as one variable, and a suitable application of Theorem 2.6 (with $w(m, n) \equiv 0, \tilde{w}(m, n) \equiv 1, \tilde{b}_{i}=b_{i}, \tilde{c}_{i}=c_{i}, \tilde{d}_{i}=d_{i}, \tilde{e}_{i}=e_{i}$ there) yields the desired result.

Remark 3. We note that the results in [5-17] are not available here to establish the analysis for Theorems 3.1-3.4. 


\section{Competing interests}

The author declares that they have no competing interests.

Received: 27 March 2011 Accepted: 30 August 2011 Published: 30 August 2011

\section{References}

1. Pachpatte, BG: On a Certain Retarded Integral Inequality And Its Applications. J Inequal Pure Appl Math 5, 9 (2004). Article 19

2. $\mathrm{Ma}, \mathrm{QH}$ : Some new nonlinear Volterra-Fredholm-type discrete inequalities and their applications. J Comupt Appl Math. 216, 451-466 (2008). doi:10.1016/j.cam.2007.05.021

3. $\mathrm{Ma}, \mathrm{QH}$ : Estimates on some power nonlinear Volterra-Fredholm type discrete inequalities and their applications. J Comupt Appl Math. 233, 2170-2180 (2010). doi:10.1016/..cam.2009.10.002

4. Deng, SF: Nonlinear discrete inequalities with two variables and their applications. Appl Math Comput. 217, 2217-2225 (2010). doi:10.1016/j.amc.2010.07.022

5. Gronwall, TH: Note on the derivatives with respect to a parameter of solutions of a system of differential equations. Ann Math. 20, 292-296 (1919). doi:10.2307/1967124

6. Bellman, R: The stability of solutions of linear differential equations. Duke Math J. 10, 643-647 (1943). doi:10.1215/S00127094-43-01059-2

7. Ou-lang, L: The boundedness of solutions of linear differential equations $y^{\prime \prime}+A(t) y^{\prime}=0$. Shuxue Jinzhan. 3, 409-418 (1957)

8. Pachpatte, BG: On some new inequalities related to a certain inequality arising in the theory of differential equations. J Math Anal Appl. 251, 736-751 (2000). doi:10.1006/jmaa.2000.7044

9. Cheung, WS, Ma, QH, Pečarić, J: Some discrete nonlinear inequalities and applications to difference equations. Acta Math Sci. 28(B):417-430 (2008)

10. Cheung, WS, Ren, JL: Discrete nonlinear inequalities and applications to boundary value problems. J Math Anal Appl. 319, 708-724 (2006). doi:10.1016/j.jmaa.2005.06.064

11. Ma, QH, Cheung, WS: Some new nonlinear difference inequalities and their applications. J Comupt Appl Math. 202, 339-351 (2007). doi:10.1016/j.cam.2006.02.036

12. Pang, PYH, Agarwal, RP: On an integral inequality and discrete analogue. J Math Anal Appl. 194, 569-577 (1995). doi:10.1006/jmaa.1995.1318

13. Pachpatte, BG: On some fundamental integral inequalities and their discrete analogues. J Inequal Pure Appl Math 2, 13 (2001). Article 15

14. Meng, FW, Li, WN: On some new nonlinear discrete inequalities and their applications. J Comupt Appl Math. 158 407-417 (2003). doi:10.1016/50377-0427(03)00475-8

15. Meng, FW, Ji, DH: On some new nonlinear discrete inequalities and their applications. J Comupt Appl Math. 208, 425-433 (2007). doi:10.1016/j.cam.2006.10.024

16. Cheung, WS, Ma, OH: On certain new Gronwall-Ou-lang type integral inequalities in two variables and their applications. J Inequal Appl. 8, 347-361 (2005)

17. Jiang, FC, Meng, FW: Explicit bounds on some new nonlinear integral inequality with delay. J Comput Appl Math. 205, 479-486 (2007). doi:10.1016/j.cam.2006.05.038

doi:10.1186/1687-1847-2011-30

Cite this article as: Zheng: Qualitative and quantitative analysis for solutions to a class of Volterra-Fredholm type difference equation. Advances in Difference Equations 2011 2011:30.

\section{Submit your manuscript to a SpringerOpen ${ }^{\circ}$ journal and benefit from:}

- Convenient online submission

Rigorous peer review

- Immediate publication on acceptance

- Open access: articles freely available online

- High visibility within the field

- Retaining the copyright to your article

Submit your next manuscript at $\gg$ springeropen.com 